\title{
DIANA - DISPOSITIVO DE ANÁLISE E CONTROLE DE ESTUFAS AGRÍCOLAS
}

\author{
Carlos Eduardo Bastos ${ }^{l}$, Thales Ferreira ${ }^{l}$, Rafael Galli ${ }^{*}$ \\ ${ }^{1}$ Departamento de Engenharia Elétrica, IFSUL Câmpus Pelotas, 55740-000, Pelotas, Brasil. \\ ${ }^{2}$ Departamento do curso Técnico em Eletrônica, IFSUL Câmpus Pelotas, 55740-000, Pelotas, Brasil.
}

*E-mail: rgalli@pelotas.ifsul.edu.br

Recebido em: $30 / 12 / 2018$ Aceito em: 08/01/2020

\section{RESUMO}

O cultivo em estufas agrícolas sem o monitoramento adequado, em função do descontrole climático, impossibilita o agricultor à produção adequada de determinados alimentos. Com base nisso, desenvolveu-se o projeto de pesquisa DIANA (Dispositivo de Análise e Controle de Estufas Agrícolas). Considerando que, o cultivo em uma estufa com o ambiente controlado apresenta melhores resultados do que o cultivo em campo aberto, é através da análise e controle do tempo que se obtêm os melhores resultados em cultivos. $\mathrm{O}$ dispositivo DIANA tem por objetivo proporcionar ao usuário um sistema eletrônico que atue na busca por garantir as condições climáticas ideais para a produção em estufas de hortifrutigranjeiros, pois simula-se um microclima desejado em seu interior. O aparelho eletrônico indica ao usuário os dados climáticos em tempo real. Para isto, o sistema conta com sensores de luminosidade, umidade relativa do solo, umidade relativa do ar e temperatura. Após efetuar toda a etapa de monitoramento, o usuário poderá selecionar as condições climáticas que deseja na central do equipamento. Depois de selecionados os valores desejados, o sistema atuará automaticamente, a fim de controlar as propriedades climáticas por meio de atuadores elétricos. O equipamento tem sido trabalhado em escala de bancada e estuda-se sua implantação em escala piloto, ou seja, uma estufa de média escala para fins de testes de germinação, tendo repetição dos mesmos tratamentos em ambiente não controlado, ou seja, mediante as intempéries, para comparação. Portanto, o cultivo controlado em estufas resolve a problemática das intempéries climáticas e propicia ao agricultor e a população um produto de melhor qualidade.

Palavras-chave: Sistema eletrônico. Condições climáticas. Cultivo controlado.

\section{Introdução}

A agricultura é um importante setor da economia brasileira, fundamental para o crescimento do país. É uma atividade altamente dependente de fatores climáticos, cujas alterações podem afetar a produtividade e o manejo das culturas, além de atingir fatores sociais, econômicos e políticos [1].

Como relatado em diversos meios, muitos produtores rurais sofrem com as variações climáticas em suas plantações, contabilizando prejuízos que geram queda na qualidade do produto e consequente aumento nos preços dos hortifrutigranjeiros, prejudicando o agricultor e a população consumidora.

O potencial impacto adverso das mudanças climáticas sobre a agricultura brasileira e a subsistência a ela associada é uma questão sobre a qual pesquisadores e produtores têm dado especial atenção. Há uma preocupação crescente com relação à hipótese de que o aumento da variabilidade climática tenha impactos negativos significativos na agricultura, no crescimento econômico nacional e nas subsistências correlatadas do Brasil [2].

Existem alterações climáticas em cada região, que ocasiona grandes impactos que dificultam o cultivo. Qualquer espécie de cultura sofre decorrente a estas variações. Principalmente com regiões muito distantes uma da outra e fatores de instabilidade do clima que encontramos hoje em dia [3].

Considerando estes fatos e que "[...] a capacidade de controlar o ambiente interno da estufa faz com que os resultados sejam melhores dos que os conseguidos em campo aberto" [4], foi desenvolvido o projeto de pesquisa DIANA - Dispositivo de Análise e Controle de Estufas Agrícolas, que pretende propiciar ao setor agrícola, desde a agricultura familiar até as agroindústrias, as condições climáticas ideais para o plantio e o desenvolvimento da safra.

O projeto teve o objetivo de construir um sistema eletrônico que proporcionasse condições climáticas ideais, garantindo maior 
seguridade e qualidade de produção. Por meio disso, o usuário poderá definir os valores ideais de umidade relativa e temperatura para o seu determinado tipo de produção. $\mathrm{O}$ dispositivo atuará monitorando as condições do ambiente e controlando-as automaticamente através de irrigadores e exaustores, a fim de mantê-las constantes.

É importante ressaltar que o equipamento apresenta características sustentáveis, como a utilização de energia solar para recarregar a bateria, tornando seu funcionamento contínuo e independente da energia oferecida pela concessionária local, além da economia de água utilizada no cultivo, uma vez que a plantação recebe somente o necessário. Quanto aos benefícios para o usuário, destaca-se a possibilidade de controlar e monitorar a estufa através de um dispositivo eletrônico portátil, o que garante um monitoramento preciso e proporciona ao agricultor uma rotina menos exaustiva, uma vez que não precisa se deslocar até o local da produção.

A eficiência do projeto foi testada através de um protótipo junto a estufa desenvolvida em escala reduzida. Assim, em um ambiente pequeno e controlado, pudemos averiguar seu funcionamento e fazer uma estimativa do seu desempenho em escala real.

\section{Parte Experimental ou Metodologia}

Pesquisas foram realizadas acerca de componentes eletrônicos utilizados no sistema. Estes foram escolhidos visando à eficiência e à acessibilidade do projeto, além da garantia de um dispositivo de baixo custo e com uma qualidade satisfatória. Conforme os tópicos abaixo, diversos testes foram realizados com tais dispositivos, a fim de ampliar o conhecimento sobre eles e analisar seus comportamentos. Além da seleção dos componentes, efetuamos estudos para nos aprofundarmos no conhecimento de cultivo em estufas. Por meio de nossas pesquisas, concluímos que os fatores necessários para obter a melhor qualidade da produção são a luminosidade, umidade relativa do solo, umidade relativa do ar e temperatura. Para realizar a análise destes parâmetros, foi necessário pesquisar sobre como coletar os dados dos sensores [5], implementar curvas de resposta e realizar diversos testes acerca de sua precisão.

\subsection{Dispositivo para pesquisas e testes}

O projeto contou com etapas de análise de parâmetros climáticos. Estes dados foram obtidos através de sensores eletrônicos ligados a um microcontrolador. Esse pode atuar de forma sequencial ou combinacional. Posto isso o sistema opera realizando de forma sequencial a aquisição do sensor, interpretação do dado, cálculo matemático, armazenamento, comparação e, por fim, se necessário tomada de uma determinada decisão [6-7]. Como citado o sistema eletrônico, através da resposta do sensor, realiza funções matemáticas para converter os dados dos sensores em unidades conhecidas dos mesmos. No entanto, alguns sensores utilizados não possuem funções matemáticas fornecidas pelo fabricante. Posto isso, fez-se necessário efetuar pesquisas e testes com estes sensores em diversos ambientes e solos. A equipe do DIANA desenvolveu um aparelho eletrônico portátil, contendo um microcontrolador programável, para executar etapas de testes com os sensores (Figura 1). Esse sistema foi fornecido também ao projeto de Produção de Substratos e Fertilizantes a Partir de Podas de Árvores que está utilizando tal dispositivo para realizar aulas práticas com alunos dos cursos técnicos do IFSUL Campus Pelotas.

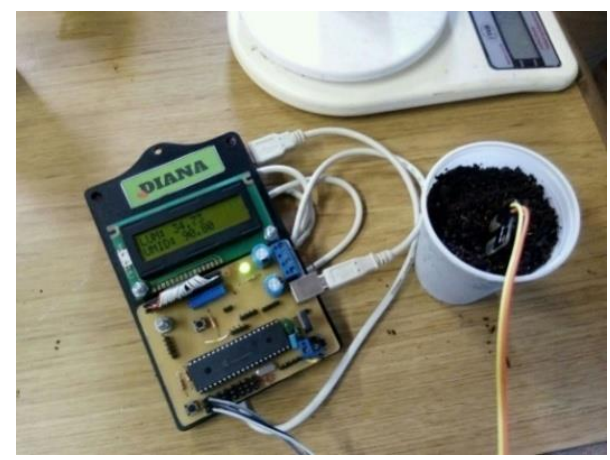

Figura 1 - Dispositivo eletrônico portátil para testes

Grande parte desses estudos foi realizado em conjunto ao projeto Produção de Substratos e Fertilizantes Orgânicos a partir de Podas de Árvores para a Produção de Alimentos e Hortaliças, no qual é constituído um núcleo de pesquisa diretamente ligado ao projeto DIANA.

Além disso, pesquisas foram realizadas acerca dos atuadores elétricos, que são os responsáveis por controlar cada propriedade climática analisada. A coleta de dados de como controlar um ambiente foi realizada em uma estufa de pequeno porte (Figura 2).

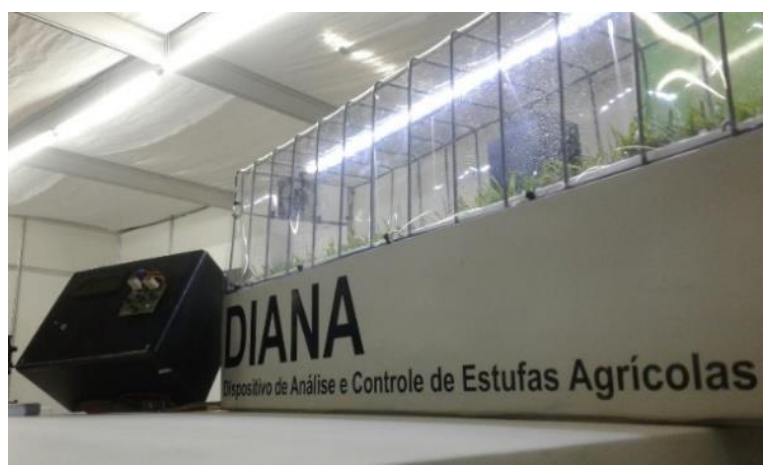

Figura 2 - Estufa de pequeno porte experimental 


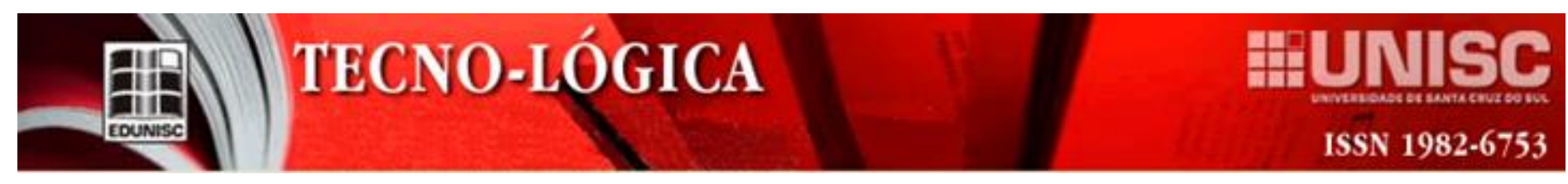

\subsection{Procedimentos utilizados}

Para realizar as pesquisas em relação às propriedades climáticas, um vasto embasamento teórico foi consultado. A fundamentação dos aspectos contemplados decorreu do exame de trabalhos similares, disponíveis por meio de relatórios técnicos emitidos por renomadas universidades do país. Assim, o referencial teórico do projeto conta com informações sobre as variações climáticas, análise climática, irrigação, luminosidade, umidade do ar, ventilação e temperatura. A partir do conhecimento teórico obtido, deu-se início à fase de pesquisa, realizada a fim de selecionar os melhores componentes eletrônicos a se utilizar no projeto. Além disso, foram estudados conceitos sobre o clima, efetuando-se sua aplicação prática no laboratório de pesquisa e inovação e laboratório de química de nossa instituição.

Com o dispositivo portátil utilizado para executar testes, iniciamos o exame do sensor de umidade relativa do solo. Fabricado na China, esse sensor teve sua compra realizada pela internet. No entanto, não foi encontrado qualquer manual sobre o novo dispositivo. Com base nisto, efetuamos pesquisas quanto ao comportamento do sensor em um laboratório de química em conjunto com o projeto Produção de Substratos. Foram realizados testes com diversos tipos de substratos orgânicos em conjunto com o sensor para descobrir seus diferentes níveis de tensão e coletar o máximo de informações possíveis para realizar a função matemática do mesmo.

Após isso, examinamos o sensor de luminosidade Light Dependent Resistor (LDR). O crescimento de uma planta por meio de iluminação artificial apresenta melhor resultado quando essa recebe a incidência correta de um fotoperíodo [8]. Assim, um sensor convencional de luminosidade como o LDR se faz necessário em função da sua praticidade de utilização e de seu custo. Apesar desse sensor não possuir manual disponibilizado pelo fabricante, não foram encontradas no texto instrucional informações relativas a aplicações para análise de lux. A padronização de medidas de luminosidade é bastante complicada devido à falta de um sistema de medição amplamente aceito [9]. Posto isso, buscou-se desenvolver um dispositivo com o LDR que atendesse às necessidades mínimas de precisão. O LDR possui a curva de resposta como uma função logaritmo (Figura 3), assim, a análise desse gráfico foi efetuada extraindo as devidas equações matemáticas para o cálculo do sensor.

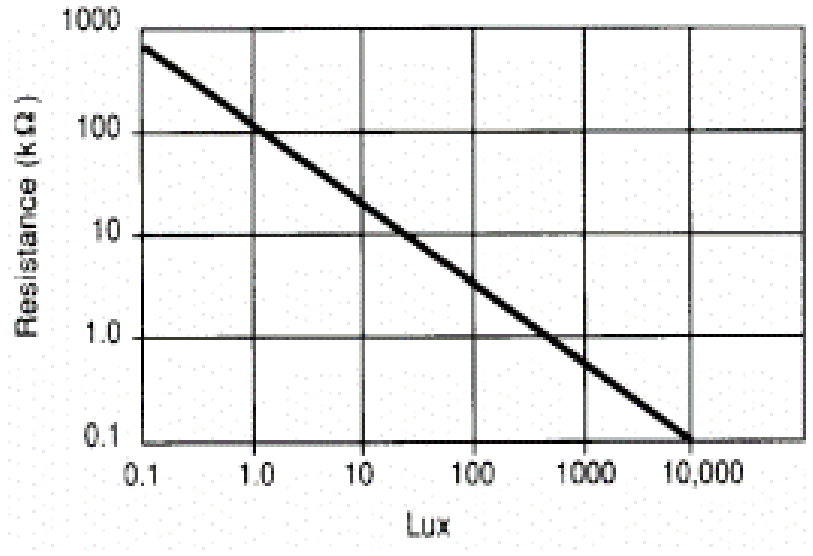

Figura 3 - Gráfico luminosidade X resistência

Com a padronização dessas medidas, testamos o dispositivo com um luxímetro profissional (Figura 4). A porcentagem de erro foi de cerca de 5\%, que é muito satisfatória para um transdutor normalmente não utilizado para fins de análise.

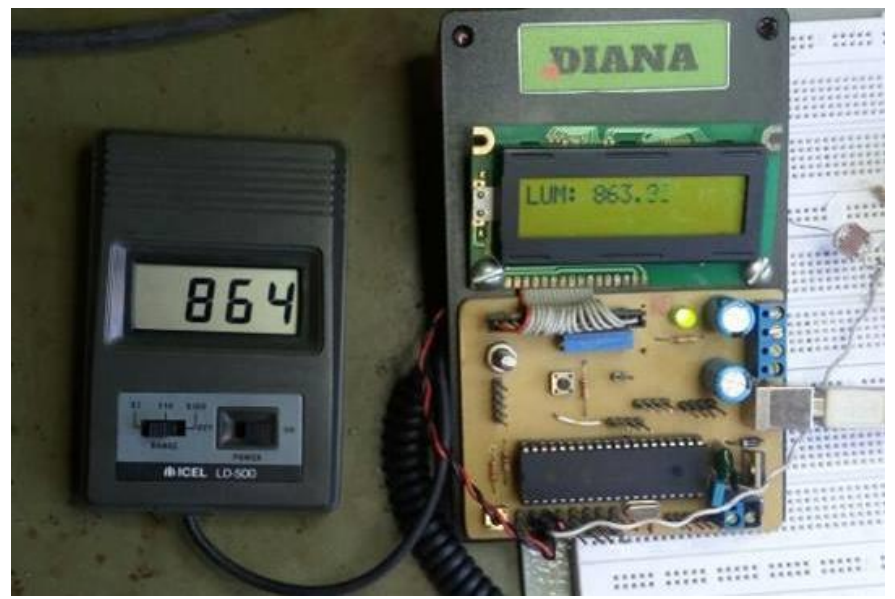

Figura 4 - Teste de eficácia com o sensor de luminosidade

O sensor de temperatura utilizado foi o LM35. Esse componente compreende temperaturas até $150^{\circ} \mathrm{C}$ e atua de forma que, a cada variação de $1^{\circ} \mathrm{C}$ na temperatura, $10 \mathrm{mV}$ variam em sua saída (Figura 5). Além disso, possuí informações muito precisas em seu Datasheet, tornando desnecessária a realização de exames avançados com o equipamento.

Os sensores de umidade relativa do solo e umidade relativa do ar também apresentam manual disponibilizado pelo fabricante com função matemática pronta. Portanto, verificações aprofundadas não foram necessárias. 


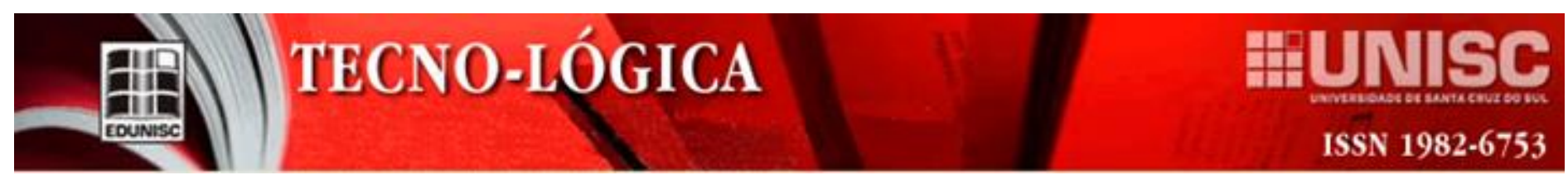

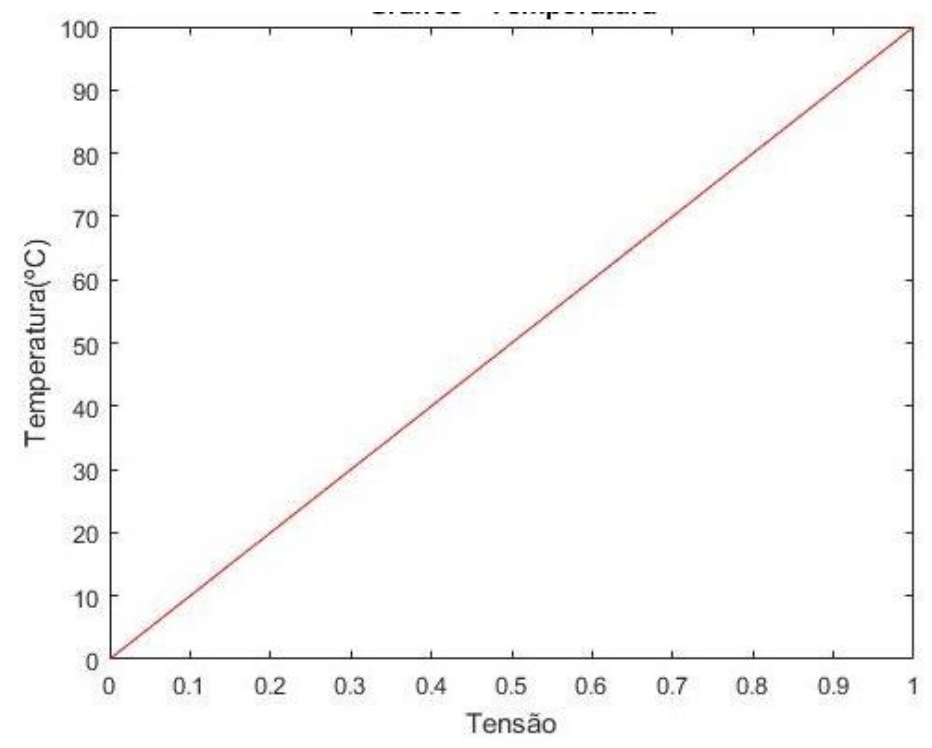

Figura 5 - Gráfico temperatura X tensão de saída do sensor

Após todas os exames com os sensores, executamos a fase de desenvolvimento do hardware e software, utilizando-se o programa Eagle para projetar os circuitos impressos e projetandose, através de técnica de transferência térmica, as placas dedicadas aos sensores, atuadores, central, dispositivo móvel e módulos de monitoramento. $\mathrm{O}$ desenvolvimento destes dispositivos foi direcionado para que o sistema fosse compatível com diversas escalas. Para analisar as condições atmosféricas em estufas de escalas maiores, tornou-se necessário apenas instalar a quantidade de módulos de monitoramento desejado, assim ampliando a área de monitoramento da estufa. Atualmente, o sistema de comunicação (RS 485) é compatível com até 32 módulos de monitoramento. Desse modo, é possível instalar o dispositivo DIANA para analisar e controlar o tempo em grandes ambientes ou até 32 pequenas estufas, sendo analisadas independentemente. Após a elaboração de todas as placas, iniciou-se o desenvolvimento do software na ferramenta CCS Compiler, para fins de programação dos microcontroladores PIC. Também, foi construído um software de análise para plataforma Windows [10], que tem conectividade com o dispositivo portátil citado alhures.

Com toda a etapa de análise climática funcionando, iniciaram-se os estudos de como controlar o ambiente através de atuadores elétricos. Como dito anteriormente, coletamos dados de vários artigos de renomadas universidades brasileiras. Este referencial teórico coletado foi a base da construção do protótipo de testes, que tem por objetivo controlar todas as propriedades climáticas analisadas. A etapa de controle é composta de um sistema de ventilação (acionada a cada 5 minutos para troca de ar dentro da estufa), sistema de aquecimento/refrigeração (constituído por uma pasta termoelétrica), sistema de irrigação (que se movimenta automaticamente dentro da estufa) atuando por meio da técnica de aspersão [11], luminosidade e umidificador do ar (composto por esponjas umidificadoras). Vale ressaltar que um sistema de irrigação que consuma pouca água e que atue de forma eficiente é essencial na produção para assim gerar benefícios ao produtor ao meio ambiente [12].

Assim, a arquitetura do dispositivo permite que o usuário insira valores desejados e o equipamento automaticamente propicia o microclima desejado. Abaixo, encontra-se a estufa para demonstração em eventos com a devida posição dos atuadores elétricos dentro de uma estufa de pequeno porte (Figura 6).

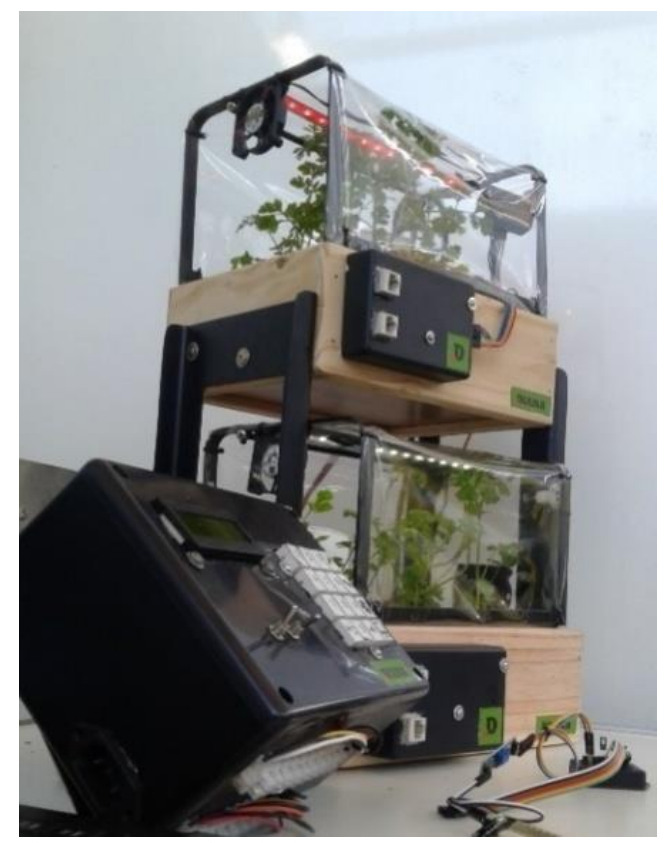

Figura 6 - Protótipo de demonstração

\section{Resultados e discussões}

Em virtude de o projeto ainda estar em fase de desenvolvimento, ainda não tivemos oportunidade de testá-lo em uma aplicação real No entanto, testes já foram idealizados e o local já foi selecionado. Inicialmente implantaremos o DIANA em uma estufa em escala reduzida, que será construída pelos integrantes da equipe. Nessa estrutura, serão cultivados determinados frutos com diferentes tipos de substratos, que foram produzidos pela compostagem em um projeto associado ao Diana em um núcleo de trabalhos institucionais agrícolas. A estufa construída consiste em um tanque de água de $1 \mathrm{~m}$ x $1 \mathrm{~m}$ reciclado, no qual serão instalados o protótipo e a requerida estrutura para o cultivo, que se resume ao módulo de monitoramento, os atuadores, vasos plásticos para o plantio e duas prateleiras para sustentação. Pode-se assimilar a estufa com uma geladeira, a qual possui prateleiras e uma 
propriedade climática diferente do meio externo. Vale ressaltar que a estufa possuirá dois exaustores que atuarão em conjunto, a fim de remover o ar viciado internamente: um deles será instalado na parte inferior, ficando responsável por efetuar a sucção do ar para a estufa; o outro, na parte superior, expelindo o ar da estufa para o meio externo. O módulo de monitoramento será fixado na parte superior da estufa, próximo ao topo, com o propósito de proporcionar uma melhor captação dos sensores.

\section{Conclusões}

Durante o desenvolvimento do projeto algumas possíveis melhorias foram observadas. Dentre esses aprimoramentos, destaca-se a possibilidade de aumentar a praticidade do projeto por meio de um aplicativo com comunicação via Wifi. Tal aplicativo possibilitará ao usuário monitorar e controlar a estufa do seu dispositivo móvel. Outra possível melhoria seria implantar a comunicação da rede dos módulos via wireless, diminuindo o custo do projeto, uma vez que o cabeamento é eliminado. No entanto, pode-se concluir que o cultivo controlado em estufas resolve a problemática das intempéries climáticas e propicia ao agricultor e a população um produto de melhor qualidade [13].

\section{Agradecimentos}

Diante da realização deste projeto gostaríamos de agradecer a todos que nos orientaram e apoiaram para que este trabalho viesse a se tornar realidade. Primeiramente agradecemos aos nossos familiares por sempre nos apoiarem em todos os aspectos, e compreenderem nossas ausências durante o processo de desenvolvimento do projeto. Ao IFSUL Campus Pelotas por viabilizar as condições materiais, bem como aos servidores e professores do IFSUL Campus pelotas pelo apoio e carinho. Em especial aos professores da COLINC - coordenadoria de linguagens do Campus Pelotas pelo apoio nas revisões e correções ortográficas. A Pró-Reitoria de pesquisa, inovação e pósgraduação do IFSUL Campus Pelotas. A todos os alunos e professores que compõem o LAB 14 pelo apoio, orientação e carinho de cada um. Ao professor Rafael Galli pelos seus ensinamentos e instruções. Ao professor Éder Coutinho pelo apoio, empenho e dedicação nas pesquisas científicas e no reconhecimento da equipe nas diversas áreas. A aluna Manuela Carrazzoni pelo apoio e dedicação nas questões gráficas e publicitárias projeto. A todos que foram mencionados e aos demais que de alguma forma estão envolvidos no processo de construção deste trabalho, só temos a deixar o nosso "Muito obrigado".

\section{DIANA - GREENHOUSES MONITORING AND CONTROL DEVICE}

ABSTRACT: Agriculture is an important sector of the Brazilian economy, fundamental for the growth of the country. Many rural producers suffer from climatic variations in their plantations, accounting for losses in production, a fall in product quality and consequent increase in the price of horticultural crops. The cultivation in agricultural greenhouses without the proper monitoring, due to the lack of humidity and temperature, makes it impossible for the farmer to produce certain foods. The DIANA project aims to provide the user with an electronic system that acts in the search to ensure ideal climatic conditions for the crop, providing a good development of the production. The device allows the user to view the weather data in real-time and the system acts by automatically controlling these properties. It has compatibility for expansion of the control and monitoring, allowing one or more greenhouses to enjoy the same system, being analyzed and controlled independently. This system has a group of sensors that perform the functions of climate analysis. Climate control is done through irrigators, exhaust fans, heating, cooling, and lighting systems. Both the monitoring and control systems are controlled through a PIC microcontroller, with algorithms and electronic circuits developed during the project. The equipment is being deployed in a medium-scale greenhouse for testing purposes by a team of students and teachers in a research nucleus. By observing the operation of the system, we intend to compare cultures performed within the controlled environment with cultivated externally. Therefore, DIANA is an easy-to-use system accessible to several producers. The device allows the user to enter the desired climatic data, providing a system compatible with various crops. A certain saving of water is emphasized since the system only uses what is necessary for production. In addition, the device does not require solar lighting for production, thus making it possible to use indoor controlled greenhouses.

Keywords: Climate analysis. Controlled environment. Electronic system.

\section{Referências}

[1] LIMA, M. A.; CABRAL, O. M. R.; MIGUEZ, J. D. G. Mudanças climáticas globais e a agropecuária brasileira.Jaguariúna, SP: EMBRAPA Meio Ambiente p. 397, 2001.

[2] PELLEGRINO, Q.; ASSAD, E. D.; MARIN, F. R. "Mudanças climáticas globais e a agricultura no Brasil". Revista Multiciência, n. 8, p. 139-162, 2007.

[3] CAMARGO, Claudia; MACHADO, Ludmila; PANDOLFO, Cristina; BRAGA, Hugo. Estudo de tendências climáticas da temperatura do ar no estado de Santa Catarina. Florianópolis, SC: Eng. Agr., Centro de Ciências Agrárias, Estudante, UFSC, 2009.

[4] MURGUERO JUNIOR, Ronaldo T. Automação de Estufas Agrícolas. In: SILVA, Juarez Bento da; BILESSIMO, Simone Meister Sommer. Anais do I Seminário de Pesquisa, Pós-Graduação e Inovação. Universidade Federal de Santa Catarina: Araranguá, SC. 2016. 
[5] PEREIRA, Fábio. Microcontroladores PIC: Programação em C. São Paulo: Érica, p. 2003.

[6] BIGNELL, James W.; DONOVAN, Robert L. Eletrônica Digital - Lógica Combinacional. São Paulo: Makron Books, Vol. 1, 1995.

[7] BIGNELL, James W.; DONOVAN, Robert L. Eletrônica Digital - Lógica sequencial. São Paulo: Makron Books, 1995. V. 2

[8] BÓSIO, Anderson; NASCIMENTO, Everton; SANTOS, Gabriel; QUEIROZ, Gustavo; CUSTÓDIO, Gustavo- Análise de crescimento de plantas de acordo com o espectro de luz e fotoperiodismo. UFAB, agosto de 2011.

[9] PINHO T. ROSVALL, E. T. M. E. P.; HALONEN, L. Light emitting diodes in plant growth: Comparative growth test in greenhouse and evaluation of photosynthetic radiation. Helsinki University of Technology, Department of Electronics - Lighting Unit, Espoo, Tech., 2008.

[10] JAMSA, Kris Ph.D. e KLANDER, Lars. Programando em C/C++. A Bíblia O melhor guia para programação em C/C++. São Paulo: Makron Books.1999.

[11] BISCARO, Guilherme A. - Sistemas de Irrigação por Aspersão. Dourados, MS: Ed. UFGD, 2009.

[12] SOUZA, Claudinei F.; MATSURA, Edson E. Avaliação de sondas de TDR multi-haste segmentadas para estimativa da umidade do solo. Revista Brasileira de Engenharia Agrícola e Ambiental, Campina Grande, v. 6, n. 1, p. 63 - 68, 2002.

[13] PAGIUCA, Larissa G., SILVA, Amanda R. e SILVA, Bruna A. Cultivo protegido - Em busca de mais eficiência produtiva. Piracicaba, SP: Ed. CAPA, 2014. 